\title{
Analysis of Social Network Dynamics with Models from the Theory of Complex Adaptive Systems
}

\author{
Ilias Lymperopoulos and George Lekakos
}

\author{
Department of Management Science and Technology \\ Athens University of Economics and Business, Athens, Greece \\ \{eliasliber, glekakos\} @aueb.gr
}

\begin{abstract}
The understanding and modeling of social dynamics in a complex and unpredictable world, emerges as a research target of particular importance. Success in this direction can yield valuable knowledge as to how social phenomena form and evolve in varying socioeconomic contexts comprising economic crises, societal disasters, cultural differences and security threats among others. The study of social dynamics occurring in the aforementioned contexts with the methodological tools originating from the complexity theory, is the research approach we propose in this paper. Furthermore, considering the fact that online social media serve as platforms of individual expression and public dialogue, we anticipate that their study as complex adaptive systems, will significantly contribute to understanding, predicting and monitoring social phenomena taking place on both online and offline social networks.
\end{abstract}

Keywords: Social dynamics, social network analysis, complex adaptive systems, online social networks, online social media, offline social networks, complex networks, complexity theory, scale free networks, statistical physics, complex adaptive systems models.

\section{Introduction}

In a society where social interactions obtain a global dimension due to the increased mobility of people and proliferation of social networking tools which keep citizens in a perpetual contact, complexity and unpredictability rises at a rate faster than ever before. In this context, the analysis and modeling of social dynamics is being redefined as new approaches are introduced in studying the social systems which become all the more less-bounded, nonlocal and complex. In such a setting, nonlinear phenomena emerge on social networks as the parameters regulating the individual's behavior can bring the system to its "tipping points" where abrupt phase transitions take place thus indicating the sensitivity of social behavior to small changes in the parameters that control the system.

As the social networks become more complex, the analytical tendencies shift from the study of static structural and locational properties to the analysis of the temporal change in their structure and composition and its impact to ties among individuals and social norms. Furthermore, new thinking concepts are applied when analyzing their 
structure as it is considered the result of a self-organization process giving rise to highly connected social clusters playing a crucial role in terms of social stability and transmission of interaction. Also, the interplay between individuals' state and social network topology causes a host of complex social dynamics such as opinion formation, spread of ideas, influence, epidemics and community formation among others.

This new theoretical framework originating from complexity theory sets the study of social dynamics on a new basis. As a result, we propose that social behavior phenomena stemming from economic crises, societal disasters, cultural differences and security threats, be studied and modeled as "emergent properties" deriving from simple forms of local social interactions spawning global effects. In that vein, trust, confidence, influence and persuasion can also be cast in complexity theory terms and modeled as outcomes of the interplay between nodes' state and social network topology. To this end, the analysis of information obtained from online social media which serve as platforms of individual expression and public dialogue, will significantly contribute to understanding, predicting and monitoring social dynamics occurring on both online and offline social networks.

The paper is organized into six sections. After the brief introduction, section two provides a review of methodologies applied to structural and locational analysis of social networks outlining the type of problems these tools are suitable for, meanwhile identifying their weaknesses in dealing with the analysis of the temporal dynamics taking place on them. Section three introduces network models appropriate for studying the co-evolutionary adaptation of network dynamics and topology in social networks. Section four focuses on significant topological properties common among a large number of complex networks and illustrates why offline and online social networks can be analyzed as complex adaptive systems. Section five presents models originating from the complex adaptive systems theory used for studying social phenomena, thus providing evidential proof of suitability for analyzing social dynamics occurring on online social networks. The paper closes with the concluding remarks in the sixth section.

\section{Structural and Locational Analysis of Social Networks}

Tracing the beginnings of the Social Network Analysis scientific field, we go back in the early 1930's when Moreno [1] introduced the sociogram - a new methodological technique which was described as "invention" since it was the first systematic attempt to plot the structure of social relations in a group. This innovation heralded the advent of sociometry and social psychology which established qualitative methods for exploring socio-emotional networks [2]. Since then, much progress has been achieved with the theoretical notions of communities, popularity, prestige, transitivity, clique, social role, reciprocity, influence, dominance and conformity, providing impulse for the development of social network analysis methods [3].

Tools from network theory have been used for the description and analysis of social networks. This methodological approach was initiated by anthropologists such as Mitchell and Barnes who noticed that the analysis and understanding of the 
people's behavior in societies of increasing complexity, as the population was moving from rural to urban areas, could not be performed under the traditional concepts of social institutions such as economics, religion, politics e.t.c. The application of network theory to social network analysis, was further expanded by Wasserman's and his colleagues research centered around the principle that social phenomena share an underlying relational network structure and therefore they can be analyzed with the theoretical framework of graph theory [2, 3].

The application of graph theory in the social network analysis field provided a powerful set of tools for describing and modeling the relational context in which behavior takes place, as well as the relational dimensions of that behavior. Graph theory was also applied in the investigation of associations among concepts and developmental phenomena such as the structure of personal life histories. It was also the basis for the development of methods addressing the identification of cohesive groups, blockmodeling and equivalence analysis, dynamical network analysis, structural balance and methods for the analysis of two mode data (e.g. person by event). Approaches like the foregoing ones rest on the capability of graph theory to systematically measure the properties of a social structure in a way that renders modeling and comparison smoother [4]. With their capacity to analyze network structural properties, the methodological tools supplied by graph theory have been successfully applied in predicting work satisfaction and team performance [5], power and influence [6], success in bargaining in a competitive context [7,8], mental health outcomes and a variety of other social phenomena. In the recent years the methodologies based on graph theory have evolved as powerful instruments for structural and locational analysis of social networks [2, 3, 9-12].

However, despite the successful application of graph theory in social network analysis tasks pertaining to their descriptive features, the analytical focus is limited to static network properties. This downside has begun to being addressed lately as the emphasis shifts from analyzing static properties to analyzing dynamic processes taking place on social networks [13].

In tandem with the shift of focus on the dynamic properties of social networks structure, efforts have been made to employ basic statistical measures of probability and significance to assess the validity of propositions about network structure [14]. However despite the analytical power of standard statistical tools such as regression, significance tests and variance analysis, their application to network data comes with drawbacks originating from the hypothesis that network observation data is independent, an assumption that contradicts with typical network data. This inefficiency has been addressed by Stanley Wasserman and his colleagues $[15,16]$ with the introduction of the Exponential Random Graph Models (often referred as $\mathrm{p}^{*}$ models). These models randomly produce graphs on a given set of points covering the entire range of possible networks from completely unconnected to fully connected. The log odds ratios of the probability of each randomly generated graph are used for producing Monte Carlo estimations that allow the comparison of a real network with the set of networks previously created by the model so that it can be estimated how possible is that the actual network can exist only by chance. This modeling technique can be used for validating theories about network structures and relational process. In other words the implemented model puts forward specific structural results which are then 
compared with the real network data taken from observations. To exemplify the concept of this modeling technique we can hypothesize that mutuality and cooperation tendencies appear frequently among groups with common interests in a community. For assessing the validity of this proposition we can construct an ERGM statistical model incorporating the hypothesis and subsequently test whether statistical results are confirmed by empirical data.

In general, the main fields of social network research concern: (i) the study of the static topological properties, (ii) the modeling of network formation (either static or growing), and (iii) how the dynamical processes occurring on networks are affected by the network topology [17].

Nevertheless, when trying to reproduce the topological properties of real networks by means of simple graph models there exist difficulties in deriving certain properties often common between networks of different nature thus indicating the existence of common organizing mechanisms [17]. The limitations -inherent in simple graph models- have been the subject of recent research focusing on the temporal aspects of networks growth as well as on the dynamics occurring on local and global level. The next section of this paper serves as a synopsis of the developments on this field meanwhile proposing new types of network models for the analysis of both offline and online social networks.

\section{Temporal Dynamics of Social Networks}

The foregoing approaches serve the structural and locational analysis of social network properties. However, for investigating the temporal network dynamics new concepts such as the small world [18, 19], preferential attachment [20, 21], percolation [22, 23] and epidemiology [24-26] are used as they better represent the structure and growth dynamics of social networks showing the following nontrivial topological properties: (i) Correlation (or anticorrelation) between degrees of neighboring nodes leading either to establishment of a link (assortativity) or the avoidance of the connection (dissartotativity) [12, 26, 27], (ii) Small World effect [18, 19] and (iii) High Clustering Coefficient [20].

Since the social network evolution is based on the co-evolutionary adaptation of dynamics and topology by local rewiring rules, a question emanating from this observation is whether co-evolution of dynamics extends to a global time scale despite the local nature of the rewiring events and a significant time scale separation between dynamical and topological updates [28]. Focusing on the interactions between the nodes of a social network rather than the attributes of the nodes themselves for deriving insights as to the dynamics taking place on social networks, an interdisciplinary perspective is being formed encompassing the scientific fields of statistical physics, nonlinear dynamics, critical phenomena, fractal geometry, spin glasses, and many-body theory [17].

It is asserted that the complexity of real-world networks stems from the interplay between the network topology and network dynamics (figure 1). Several studies up until now concentrate either on the effect that topological properties have on network 
dynamical processes or the opposite, that is the effect of node-specific dynamical variables on network structure [17]. As a result an interesting line of research is the investigation of the combined effect of these processes on each other. Such an approach entails that we should examine the network dynamical processes and the network growth as happening in the same timescale and not on separated ones in which case the slower variables enslave the faster ones. The simultaneous execution of these processes give rise to a new class of networks which are described with the term Complex Adaptive Systems.

According to the definition provided by G.M. Murray, "A complex adaptive system acquires information about its environment and its own interaction with that environment, identifying regularities in that information into a kind of 'schema' and acting in the real world on the basis of that schema" [29]. This means that a Complex Adaptive System (CAS) derives from its surrounds which at the same time is affected by the CAS itself. The complexity of these systems is attributed to the dynamic nature of interactions among the network nodes giving rise to system properties which cannot be handled as aggregations of the properties of the individual static entities. These systems are adaptive because the individual and collective behavior mutates and self-organizes in response to a triggering micro-event or series of events.

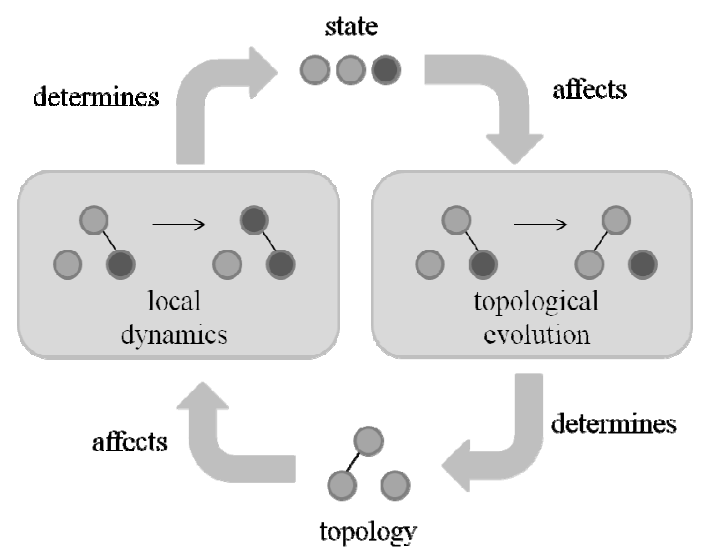

Fig. 1. In an adaptive network the global topology and the dynamics of the nodes are coupled with a feedback loop[30]

The complexity of a social network results from the dynamic feedback loops inherent in the human behavior in a societal context. These feedback loops cause the system to behave nonlinearly. It is really astonishing how fast a social network can pass to the complexity realm. The connections among individuals who predict and react to the predictions and actions of the others coupled with the progressive exacerbation of these actions as the system becomes all the more connected, leads to nonlinear interactions difficult to decompose and then complexity sets in [31]. For instance, in the opinion formation process, the beliefs of each individual node of the social network, and the evolution of the topology of the network are blended in a way that people's 
connectivity affects their opinions and at the same time their opinions affect their connections [32].

The adaptive dynamics is introduced in a social network due to the ability of the individuals to control the number, type and duration of their interactions with others [33]. After a large number of adaptive cycles, evolution towards a self-organized critical state is observed. An adaptive coevolutionary scheme leading to network selforganization is based on the theory that correlated activity connects, whereas decorrelated activity disconnects [28]. This coevolutionary pattern is also found in the neural network theory which posits that if the activity of two neighboring neurons is on average highly correlated then they will obtain a common link. However if their activity is on average less correlated, then they will lose their common link [28]. The Hebbian learning shows that network self-organization by correlation-driven rewiring is robust even when spatial constraints are present and dynamics is affected by noise [34]. The phenomenon of self organized criticality constitutes an important feature of adaptive networks and boils down to the fact that each node in a network is recipient of dynamical information which relates to the connectivity of the entire network, thereby providing global information to individual nodes thus causing the network to organize itself [17, 28].

Social networks are self-organized systems in that they consist of many interacting entities co-operating for achieving the desired result. The structure of a selforganizing system emerges without explicit external force and is the result of internally enforced constraints generated by the interactions among the entities. A self-organized system evolves temporally and spatially thus exhibiting varying organizational structures. The actions taking place in a self-organized system result from the negative and positive feedback mechanisms by means of which the system gears towards stability or chaos respectively [31].

Adaptive social networks result from the combination of contact processes with rules for network topological evolutions. Contact processes refer to how individuals are changed and shaped through interactions with others. For example, present models study the information spreading as a result of two rival process that is social adjustment (contact process) and social segregation (topological evolution) [35]. Holme and Newman report that the diversity of opinions coexisting in a society go through a phase transition if the relative rate of social adjustment and social segregation crosses a critical value [36]. When social segregation is coupled with social adjustment this leads to an adaptive network [35].

As complex adaptive systems social networks have to cope with the contradictory needs of accommodating response to changing environmental signals, while maintaining a sufficient level of stability in the dynamical networks that process this information. This remark gives rise to the idea that complex adaptive systems may have evolved to the "edge of chaos" between ordered and disordered dynamical regimes [37].

The consideration of adaptive social networks is an important step towards more realistic models of social interactions in structured populations. Coupling the dynamics on networks with the dynamics of networks leads to emergent new phenomena outside the classical context of social dynamics on static networks [33]. However a 
complete understanding of the behavior of adaptive networks requires tools which encompass stochastic dynamics, topology and time dependent graph theory [38].

\section{The Structure of Social Networks}

Over the past years researchers have found that a large number of complex systems share some important common properties which hold a prominent role in the way complex systems are studied [20]. In brief these properties refer to:

Small worlds: This concept outlines the fact that despite the large size of complex networks, the path between any two nodes is relatively short [18].

Clustering: It is often observed in complex networks that there are fully connected sub-graphs. In social networks this is called "cliquishness" and refers to circles of friends where each individual knows each other. The propensity of social networks to cluster is quantified by the clustering co-efficient metric $[18,19]$.

Scale free structure: The degree distribution shows a scale free structure that is a heterogeneous network topology encompassing a relatively small number of nodes with exceptionally high degree and a very large number of nodes with low degree (figure 2). One remarkable feature of scale-free networks is that they are highly robust against random errors but on the contrary they are highly vulnerable to attacks targeting nodes which serve as hubs in the network. The scale free modeling lays the emphasis on the network dynamics and its main objective is to model the process that underpins the evolution of complex networks since such an approach will lead to the creation of networks with the correct structure [20, 25].

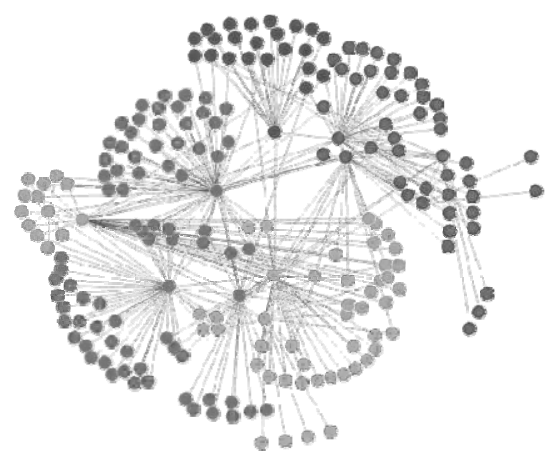

Fig. 2. Scale Free network: Small number of nodes with high degree and a large number of nodes with low degree

The aforementioned features have been observed in a remarkable number of networks including the internet, the World Wide Web, scientific collaboration, actors, citation, business, molecular, biological and social networks among others [39, 40]. Recently, empirical results show that online social networks encompass the foregoing 
properties. Mislove et al. [41] studied the online social networks Flickr, LiveJournal, Orkut and Youtube and their results show that these networks show the small world, high clustering and scale free properties observed in offline social networks. Catanese et al. [42] studied the Facebook friendship relations, and discovered that Facebook also comprises the small world, high clustering and scale free properties featured in offline social networks. These studies confirm that online social networks contain a large, densely connected cluster of high-degree nodes, bordered by lots of small clusters of low-degree nodes. This implies that high-degree nodes are crucial for the connectivity network and the flow of information which in social systems is content dependent, meaning that different type of information may be spreading over different social networks adhering to different dynamical rules [43].

From the above and similar considerations, becomes evident that an in-depth understanding of the social dynamics occurring on online social networks can yield significant insights as to how similar social phenomena take their course on off-line social networks. As a result, the analysis of online social networks from the perspective of Complex Adaptive Systems emerges as an innovative approach by means of which social phenomena can be better understood and modeled with wide scale socioeconomic benefits as predictability and monitoring power is gained regarding social transformations brought about by economic crises, disasters, security threats, criminality, epidemics and globalization to name but a few pressing issues. In this theoretical framework the study of the intermediate-scale substructures in networks, consisting of vertices more densely linked to each other than the rest of the network, has become an outstanding research subject in the complex network theory [44].

Models originating from the Complex Adaptive Systems theory which can be used for effecting the foregoing research objectives are presented in the next section. These models are able to capture the dynamic nature of social phenomena thus providing distinctive analytical advantages when it comes to dealing with the temporal aspects of interactions in social networks consisting of heterogeneous actors.

\section{$5 \quad$ Studying Social Phenomena with Complex Adaptive Systems Models}

The traditional models of studying social networks concentrate on static, homogeneous situations comprising few or a large number of agents in an environment where time and space are not of importance. However, studying the social networks with static models falls short of capturing the dynamic nature of interactions. Also, a key driving force in social networks is heterogeneity which gives rise to a rich set of alternative behaviors. With that said, traditional social networks analysis focusing on average behaviors, not only might be incomplete, but also illusory [31]. In order to overcome these shortcomings, a new category of network models has been developed with a view to capturing in a more sufficient way the complexity of many physical systems including the social ones (table 1). In the complex adaptive systems models, the topology changes dynamically in reaction to changing node characteristics and as a result the topological changes trigger changes in the dynamics occurring on nodes. 
The main characteristic of complex adaptive networks is the interplay between node dynamics and network topology meaning that nodes and links evolve over time even when a steady state is reached [45]. The scientific field of complex adaptive systems by being able to explore the dynamical aspects of the systems, is relevant for analyzing some of the most urgent social issues such as economic crises, globalization, sustainability, fighting terrorism and preventing epidemics [31].

Table 1. The new Social Network Analysis concepts focus on the dynamic nature of interactions and the heterogeneity of agents

\begin{tabular}{cc}
\hline \multicolumn{2}{c}{ Social Network Analysis } \\
\hline Traditional Models & CAS Models \\
Study of Static Situations & Study of Dynamic Situations \\
Homogeneous agents & Heterogeneous Agents \\
\hline
\end{tabular}

Studying social networks under the lens of complex networks theory entails the application of modeling techniques complying with the concept that the network topology develops by means of a feedback mechanism which connects topology and dynamical processes running on the network itself. Deciphering the network structure is actually an indispensable step in investigating dynamical processes occurring on social networks. For example, It has been shown that the network structure plays a vital role in the dynamics of ideas spread, innovations and computer viruses [46, 47].

A result of particular interest as to the spread of the diseases and how this process relates to the network structure is provided by the research conducted by PastorSatorras and Vespignagni who studied the disease diffusion dynamics on random and scale-free networks. Their research showed that while in random networks a local infection contaminates the whole network only when the spreading rate exceeds a threshold limit, in scale free networks this threshold is zero meaning that the entire population of the network will be infected [25, 48].

When modeling and studying complex systems such as social networks which comprise a large number of elements with all of their details, it is vital that the dynamics of each element and the relations among them be clarified. Network topology and nodes states often are coupled critically in such a way that the nodes behavior is constrained by the structure which at the same time is generated by the behavior of the nodes. This kind of interaction generates unpredicted behavior which is called emergence and cannot be explained at the level of elements [49].

\subsection{Statistical Physics Models}

Valuable insights into the analysis of the behavior of complex networks with applications to social ones come from the field of the statistical physics. This scientific area provides for a large number of tools catering to the prediction of a system behavior by observing the properties of its elements, e.g. how magnetism appears out of the collective behavior of millions of atoms and the regulating factor of temperature [20]. Based on this observation it could be interesting to investigate the potential existence 
of temperature-like parameters which when tuned to a critical value could lead to the formation of leaderless or hierarchically structured communities [17]. Following such an approach in the modeling of social networks could facilitate the examination of the effects of external signals or perturbations on the topology and dynamics of social networks (network-environment interaction). In this modeling scenario it could be observed that a social system reacts macroscopically even to a microscopic external perturbation thus demonstrating a behavior characterizing critical phenomena in which a dissipative system tends to rearrange itself in a way that enables the development of long-range temporal and spatial correlations (sand pile model) [31]. While many studies explore the effect of a network's nodes interaction in relation to a network's evolution, the case of network-environment interaction is largely unexplored as to the peculiarities of information processing in self-organised critical networks and the idea of finest adaptation at the "edge-of-chaos" [28].

For the study of the opinion dynamics on an adaptive random network, models from statistical physics have been successfully used thus explaining phenomena such as opinion formation, voting preferences, information diffusion and spread of diseases. Phenomena like these include a large number of "agents" which evolve over time due to the existence of external influence and noise. The final states emerge as a result of nonlinear dynamics, critical phenomena and phase transitions the outcome of which depends on the initial states and driving forces [32].

\subsection{Evolutionary Models}

Evolutionary models can be particularly helpful in examining the mechanisms underlying the growth of social networks. Bak-Sneppen model for instance, explores the feedback mechanism between fitness dynamics and topological restructuring. According to this model the node with the lowest barrier is the first to evolve as a result of interactions with the neighboring nodes. However the mutation of one node changes the state of all the interacting nodes thus affecting their fitness. When the aforementioned procedure is repeated the social network self-organizes itself in a critical stationary state in which all the barriers are distributed over certain threshold value. Given that socio-economic systems display a strongly networked structure the foregoing model could be used for examining the interactions between socio-economic nodes be they individuals, firms or trading countries [17].

\subsection{Threshold Models}

Valuable insights into how diffusion takes place in online networks is found in the in the work of Goel et al. [50]. Their paper discusses how Granovetter [51] and Lopez et al. [52] have modeled adoption decisions -in particular the expensive ones - using the "threshold" concept whereby adoption occurs only after a number of individuals (the threshold value) have adopted. Dodds and Watts [53] have proposed a model of "generalized contagion" that combines disease spread dynamics and threshold models. Young introduced a model of observational learning which resembles the threshold behavior [54]. These models demonstrate that the local and global structure of a 
social network can substantially affect the size and the probability of cascades initiated by any given seed. Many adoption models have shown that a cascade triggered by a single seed can cover the entire network provided that the circumstances are proper - as for example in forest fires which in order to be large require the proper combination of favorable factors including wind, temperature, humidity and inflammability of trees. By the same token, cascades of social influence require the appropriate combination of many factors in order to spread throughout the network thus indicating the chaotic nature of the spread dynamics, since small differences in the initial conditions could have disproportionately large outcomes. However, one important aspect that has to be taken into consideration when studying the social influence dynamics, is that the social network nodes might be highly inhomogeneous in terms of their inherent thresholds. Nodes with high thresholds are slow and switch their state only for a limited set of input configurations (similar to the effect of canalizing functions in random boolean networks). On the contrary nodes with low thresholds are more likely to switch [28].

\subsection{Neural Networks Models}

The identification and modeling of the mechanisms that govern the topological development of social networks could be enabled by introducing approaches applied in the neural networks governed by the Hebbian rule suggesting that links are reinforced between neurons being at similar states [34]. Gross' and Blasius' [30] survey regarding adaptive coevolutionary networks, provides two different approaches in studying them. The first focuses on the values of important topological properties of evolving networks and the second examines how the functioning of the network hinges on these properties.

\subsection{Cellular Automata Models}

Another approach to studying the dynamics of adaptive social networks is the use of cellular automata [49]. In these models cells with discrete states are placed on lattice topology and their state transition is controlled by the states of its neighbors. Sayama and Laramee in their survey of modeling methods for complex systems present the "Generative Network Automata" - a novel modeling technique which integrates the nodes states transitions and the network topology transformations of complex systems [55].

\subsection{Adaptive Voter Models}

Opinion formation dynamics developed on social networks could be studied by using adaptive voter models where the network topology coevolves with the state of the nodes. This model despite its simplicity can also be effective when applied to more complex systems in which a number of different opinions interact by a pairwisesymmetric competition [56]. Benczik et al [32], in their study about opinion dynamics on adaptive networks propose Voter and Voterlike models for analysing phenomena 
stemming from human behavior such as the emergence of collective organisation in socio-cultural situations. Voter model has also broad applications in the sociophysics field where in combination with statistical physics tools like mean-field approaches or numerical simulations has been broadly used for studying the spreading of cultures, religions, languages or political opinions. The Axelrod and Sznajd [57] models are particularly developed for studying the specific types of opinion dynamics.

\subsection{Molecular Network Models}

A new type of analysis applied in social networks comes from the field of biology and especially the analysis of molecular networks. This kind of analysis focuses on the discovery of local patterns in a network, called motifs [26]. Motifs are actually overrepresented small sub-graphs existing in a network. Similarly activity motifs are overrepresented patterns in the attributes of nodes and network edges.

\section{$5.8 \quad$ Agent-Based Models}

Social dynamics can also be studied by means of agent-based models which can simulate how the individual behavior leads to global transformations of network structure $[13,58,57]$. In this modeling technique, the actions of the agents which can be either individuals or groups following specific behavioral rules, are intertwined thereby affecting the overall network since the actions of each agent have a defining impact on the actions of the others. In this respect, changes in the network structure can be predicted on the basis of prior knowledge of the agents actions rules [14].

\subsection{Social Percolation Models}

With social adaptive networks encompassing the properties of "small world", "high clustering coefficient" and "power law" degree distribution [20, 59, 60], the study of network dynamics should be combined with the topological network connectivity features which can give rise to emergent phenomena of particular interest. A theoretical framework with the ability to extract global network properties from the local specifications is that of percolation which as theory lies at the crossroads of probability theory and topology [61]. A system percolates when an adequate part of its constituent elements is connected locally so that a global connection emerges.

By applying percolation theory to marketing we could explain why some products succeed and other fail. According to percolation theory if globally connected clusters do not emerge as the information about the product passes among consumers, then the commercial success of the product will be limited as there will be consumer clusters which will never be exposed to the product and as a result will never adopt it [62].

Percolation models are suitable tools for studying the emergence of phase transitions, that is a sudden and striking change in the qualitative behavior of a system triggered by a subtle change in the initial conditions. Percolation models could also be used for predicting whether a large-scale diffusion will take place or not as the 
propagation may vanish before a crucial fraction of the system is reached by the diffusion dynamics [62].

The shift to the percolation regime is usually sudden and the values of the control parameters at which the transition takes place are called "critical values". For example, adjusting this control parameters over and below the critical values can make the difference between the commercial failure and success of a product. Mort [63] proposed the application of percolation theory to marketing. Solomon and Weisbuch [64] cast the diffusion of information, beliefs, products and behavior in percolation terms thus introducing the term "Social Percolation". The "Social Percolation" framework shapes the internal social forces as local interactions between neighboring nodes and monitors the resulting adoption patterns.

\subsection{Summary of CAS Models for Analyzing Social Dynamics}

The foregoing approaches originating from the Complex Adaptive Systems theory, are summarized in table 2 associating types of CAS models and the social phenomena each of them is mostly suitable for analyzing.

Table 2. CAS models for analyzing social phenomena

\begin{tabular}{|c|c|}
\hline \multicolumn{2}{|c|}{ Complex Adaptive System Approaches for Analyzing Social Dynamics } \\
\hline Models & Social Phenomena \\
\hline Statistical Physics Models & $\begin{array}{l}\text { Opinion Formation, } \\
\text { Voting Preferences, } \\
\text { Information Diffusion, } \\
\text { Spread of Diseases }\end{array}$ \\
\hline Evolutionary Models & Growth of Social Networks \\
\hline Threshold Models & Adoption Decisions \\
\hline Neural Network Models & $\begin{array}{l}\text { Topology Changes } \\
\text { in Social Networks }\end{array}$ \\
\hline Cellular Automata & $\begin{array}{c}\text { State Transitions } \\
\text { of nodes }\end{array}$ \\
\hline Adaptive Voter Models & $\begin{array}{c}\text { Opinion Formation Dynamics, } \\
\text { Collective Organization } \\
\text { in Socio-Cultural Situations, } \\
\text { Spreading of Cultures, Religions, Lan- } \\
\text { guages, Political Opinions }\end{array}$ \\
\hline Molecular Network Models & $\begin{array}{l}\text { Activity Motifs } \\
\text { (over-represented patterns of behavior) }\end{array}$ \\
\hline Agent-Based Models & $\begin{array}{l}\text { Global Social Transformations resulting } \\
\text { from Individual Behavior }\end{array}$ \\
\hline Social Percolation Models & $\begin{array}{l}\text { Diffusion of Information, Beliefs, } \\
\text { Products, Behavior }\end{array}$ \\
\hline
\end{tabular}




\section{Conclusion}

We live in a world of networks. Communication networks, the internet, protein networks, chemical reaction networks, transportation networks, power grids, neural networks and social networks are some typical examples. However, although networks are ubiquitous their properties, structures and dynamics are not fully understood, thereby rendering the prediction of their behavior an intriguing and challenging research endeavor.

The increasing size of social networks coupled with the perpetual contact introduced by the omnipresent social networking tools, renders the social dynamics ever more complex and unpredictable. Finding the forces that drive the social transformations in a context of an overwhelmingly complex world affected by economic crises, business competition, societal disasters and security threats is a step towards gaining predictability power. To this end the study of online social networks from the viewpoint of complex adaptive systems can provide significant insights concerning social network dynamics. The methods and models presented in this paper can substantially contribute to the application of complexity theory in the study of social phenomena. Having answers as to how users build, grow, merge and break up communities, how influence is spread, how trust is built and interaction is transmitted, we will be able to explain why local events can potentially be global events with wider socioeconomic impacts.

\section{References}

1. Moreno, J.L.: Sociogram and sociomatrix. Sociometry 9, 348-349 (1946)

2. Wasserman, S., Faust, K.: Social network analysis: Methods and applications. Cambridge University Press (1994)

3. Wasserman, S., Galaskiewicz, J.: Advances in social network analysis: Research in the social and behavioral sciences. Sage Publications, Incorporated (1994)

4. Butts, C.T.: Social network analysis: A methodological introduction. Asian Journal of Social Psychology 11, 13-41 (2008)

5. Bavelas, A., Barrett, D.: An experimental approach to organizational communication. American Management Association (1951)

6. Brass, D.J.: Power in organizations: A social network perspective. Research in Politics and Society 4, 295-323 (1992)

7. Burt, R.S.: V The Social Structure of Competition (1992)

8. Willer, D.: Network exchange theory. Praeger Publishers (1999)

9. Knoke, D., Kuklinski, J.H.: Network analysis: basic concepts. In: Thompson, G., et al. (organizadores) Markets, Hierarchies and Networks, pp. 173-182. Sage Publications, London (1991)

10. Burt, R.S.: Toward a structural theory of action: network models of social Structure, Perception, and Action (1982)

11. Scott, J.: Social network analysis: A handbook. Sage Publications Limited (2000)

12. Newman, M.E.J.: The structure and function of complex networks. SIAM Review 45, 167-256 (2003) 
13. Hui, C., Goldberg, M., Magdon-Ismail, M., Wallace, W.A.: Simulating the diffu-sion of information: An agent-based modeling approach. International Journal of Agent Technologies and Systems (IJATS) 2, 31-46 (2010)

14. Scott, J.: Social network analysis: developments, advances, and prospects. Social Network Analysis and Mining 1, 21-26 (2011)

15. Wasserman, S., Pattison, P.: Logit models and logistic regressions for social networks: I. An Introduction to Markov Graphs and p. Psychometrika 61, 401-425 (1996)

16. Pattison, P., Wasserman, S.: Logit models and logistic regressions for social networks: II. Multivariate Relations. British Journal of Mathematical and Statistical Psychology 52, 169-193 (1999)

17. Caldarelli, G., Garlaschelli, D.: Self-organization and complex networks. Adaptive Networks, 107-135 (2009)

18. Watts, D., Strogatz, S.: The small world problem. Collective Dynamics of Small-World Networks 393, 440-442 (1998)

19. Watts, D.J.: Networks, dynamics, and the small-world phenomenon 1. American Journal of Sociology 105, 493-527 (1999)

20. Albert, R., Barabási, A.L.: Statistical mechanics of complex networks. Reviews of Modern Physics 74, 47 (2002)

21. Barabási, A.L., Albert, R.: Emergence of scaling in random networks. Science 286, 509512 (1999)

22. Stauffer, D., Aharony, A.: Introduction to percolation theory. CRC (1994)

23. Newman, M.E.J., Watts, D.J.: Scaling and percolation in the small-world network model. Physical Review E 60, 7332 (1999)

24. Pastor-Satorras, R., Vespignani, A.: Epidemics and immunization in scale-free networks. arXiv preprint cond-mat/0205260 (2002)

25. Pastor-Satorras, R., Vespignani, A.: Epidemic dynamics in finite size scale-free networks. Physical Review E 65, 035108 (2002)

26. Dorogovtsev, S.N., Mendes, J.F.F.: Evolution of networks. Advances in Physics 51, 10791187 (2002)

27. Caldarelli, G., Vespignani, A.: Large Scale Structure and Dynamics of Complex Networks: From Information Technology to Finance and Natural Science (Complex Systems and Interdisciplinary Science) (2007)

28. Rohlf, T., Bornholdt, S.: Self-organized criticality and adaptation in discrete dynamical networks. Adaptive Networks, 73-106 (2009)

29. Murray, G.M.: The quark and the jaguar: Adventures in the simple and the complex. Published by Little, Brown and Company (UK) Limited, London (1994)

30. Gross, T., Blasius, B.: Adaptive coevolutionary networks: a review. Journal of the Royal Society Interface 5, 259-271 (2008)

31. Miller, J.H., Page, S.E.: Complex adaptive systems: An introduction to computa-tional models of social life. Princeton University Press (2007)

32. Benczik, I.J., Benczik, S.Z., Schmittmann, B., Zia, R.K.P.: Opinion dynamics on an adaptive random network. Physical Review E 79, 046104 (2009)

33. Traulsen, A., Santos, F., Pacheco, J.: Evolutionary games in self-organizing populations. Adaptive Networks, 253-267 (2009)

34. Hebb, D.O.: The organization of behavior. Wiely, New York $(1949,2002)$

35. Do, A.L., Gross, T.: Contact processes and moment closure on adaptive networks. Adaptive Networks, 191-208 (2009)

36. Holme, P., Newman, M.E.J.: Nonequilibrium phase transition in the coevolution of networks and opinions. Physical Review E 74, 056108 (2006) 
37. Kauffman, S.A.: The origins of order: Self-organization and selection in evolution. Oxford University Press, USA (1993)

38. Shaw, L., Schwartz, I.: Noise induced dynamics in adaptive networks with applications to epidemiology. Adaptive Networks, 209-227 (2009)

39. Barabási, B.Y.A.L., Bonabeau, E.: Scale-Free. Scientific American (2003)

40. Faloutsos, M., Faloutsos, P., Faloutsos, C.: On power-law relationships of the internet topology. ACM SIGCOMM Computer Communication Review, 251-262 (1999)

41. Mislove, A., Marcon, M., Gummadi, K.P., Druschel, P., Bhattacharjee, B.: Measurement and analysis of online social networks. In: Proceedings of the 7th ACM SIGCOMM Conference on Internet Measurement, pp. 29-42 (2007)

42. Catanese, S., Meo, P., Ferrara, E., Fiumara, G., Provetti, A.: Extraction and analysis of facebook friendship relations. Computational Social Networks, 291-324 (2012)

43. Ghoshal, G.: Structural and dynamical properties of complex networks (2009), http://141.213.232.243/handle/2027.42/64757

44. Palla, G., Pollner, P., Barabási, A.L., Vicsek, T.: Social group dynamics in networks. Adaptive Networks, 11-38 (2009)

45. Gross, T., Sayama, H.: Adaptive Networks, pp. 1-8 (2009)

46. Coleman, J., Katz, E., Menzel, H.: The diffusion of an innovation among physicians, Sociometry 253-270 (1957)

47. Valente, T.W., Davis, R.L.: Accelerating the diffusion of innovations using opinion leaders. The Annals of the American Academy of Political and Social Science 566, 55-67 (1999)

48. Pastor-Satorras, R., Vespignani, A.: Epidemic spreading in scale-free networks. Physical Review Letters 86, 3200-3203 (2001)

49. Tomita, K., Kurokawa, H., Murata, S.: Graph-rewriting automata as a natural extension of cellular automata. Adaptive Networks, 291-309 (2009)

50. Goel, S., Watts, D.J., Goldstein, D.G.: The structure of online diffusion networks. In: Proceedings of the 13th ACM Conference on Electronic Commerce, pp. 623-638 (2012)

51. Granovetter, M., Soong, R.: Threshold models of diffusion and collective behavior. Journal of Mathematical Sociology 9, 165-179 (1983)

52. Lopez-Pintado, D., Watts, D.J.: Social influence, binary decisions and collective dynamics. Rationality and Society 20, 399-443 (2008)

53. Watts, D.J., Dodds, P.S.: Influentials, networks, and public opinion formation. Journal of Consumer Research 34, 441-458 (2007)

54. Young, K.S.: Internet addiction: The emergence of a new clinical disorder. Cyber Psychology \& Behavior 1, 237-244 (1998)

55. Sayama, H., Laramee, C.: Generative network automata: A generalized frame-work for modeling adaptive network dynamics using graph rewritings. Adaptive Networks, 311332 (2009)

56. Demirel, G., Prizak, R., Reddy, P.N., Gross, T.: Opinion formation and cyclic dominance in adaptive networks. arXiv preprint arXiv:1011.1124 (2010)

57. Axelrod, R.: The complexity of cooperation: Agent-based models of competition and collaboration. Princeton University Press (1997)

58. Helbing, D., Balietti, S.: How to do agent-based simulations in the future: From modeling social mechanisms to emergent phenomena and interactive systems design. Tech. Rep. 1106-024, Santa Fe Institute, NM, USA (June 2011), santa Fe Working Paper (2011)

59. Pastor-Satorras, R., Rubí, M., Diaz-Guilera, A.: Statistical mechanics of complex networks. Springer (2003) 
60. Newman, M., Barabasi, A.L., Watts, D.J.: The structure and dynamics of networks. Princeton University Press (2011)

61. Hunt, A., Ewing, R.: Percolation Theory: Topology and Structure. Percolation Theory for Flow in Porous Media, 1-36 (2009)

62. Erez, T., Moldovan, S., Solomon, S.: Social Anti-Percolation and Negative Word of Mouth. arXiv preprint cond-mat/0406695 (2004)

63. Mort, J.: Perspective: the applicability of percolation theory to innovation. Journal of Product Innovation Management 8, 32-38 (1991)

64. Solomon, S., Weisbuch, G., de Arcangelis, L., Jan, N., Stauffer, D.: Social percolation models. Physica A: Statistical Mechanics and its Applications 277, 239-247 (2000) 